\title{
PAGES Focus 4: Past Human-Climate-Ecosystem Interactions (PHAROS) Workshop
}

\section{London, UK, 7-8 July 2008}

\author{
John Dearing ${ }^{1}$ and Rick Battarbee ${ }^{2}$ \\ 'University of Southampton, UK; J.Dearing@soton.ac.uk \\ University College London, UK
}

Focus 4 (PHAROS) was formally adopted at the PAGES Scientific Steering Committee meeting in Atherton, Australia, August 2007. Its principal objective is to bring together existing and new research communities to examine how climate, natural ecosystems and human activity have interacted through time, both regionally and globally, and how these interactions help address key questions about future global environmental change.

The aims of this workshop were twofold: (1) To finalize the PHAROS Science and Implementation Plan and produce a work plan for the next five years, and (2) To coordinate the production of synthesis papers on the integration and upscaling of paleodata for key ecological processes (land cover, soil and sediment, and water), and for IPCC projected "Climate Change Hotspots". The intention was also to produce a position paper reviewing paleoperspectives on global environmental change that highlights the role of paleodata in Earth System science, current levels and coverage of reconstructed data, and future challenges for developing new methods and improving geographical representation.

35 scientists from Africa, Asia, Australasia, Europe and North America, representing the Focus 4 Working Groups of HITE, LUCIFS, and LIMPACS, and the PAGES International Project Office, met at the Royal Society to discuss how Focus 4 should progress. Following opening plenary talks from John Dearing, Marie-Jo Gaillard, Thomas Hoffmann, Peter Gell and Rick Battarbee, the meeting was structured around break-out sessions.

The first session addressed the following questions as the basis for the position paper:

- How can your scientific community best interact with or make a contribution to the research communities concerned with contemporary Earth System science?

- What are the key questions that the paleoscience community should pursue in future?

The second set of sessions dealt with regional-global syntheses, in particular:

- What can be done with current information to generate global syntheses?
- What kind of information can be upscaled?

- How can data be pooled and made available?

- How can temporal change be visualized on (global) maps?

- Are there good examples to use or follow?

- What are the priority syntheses for the future?

The second morning addressed how the different sub-disciplines could integrate results within regional landscapes, with discussion groups on four generic landscapes (monsoonal/low latitude, dryland, temperate zone, high latitude/high altitude) tasked with discussing the questions:

- How can LUCIFS, HITE and LIMPACS groups work together to provide an integrated assessment of modern global environmental change issues within a region? Which themes can be tackled by such integration?

- What were the human-ecosystem-climate interactions in the past that provide lessons for the future?

Synthesis papers were planned in the subsequent writing session. The final plenary session considered the progress made and PHAROS structure. The overwhelming view was to modify the struc-

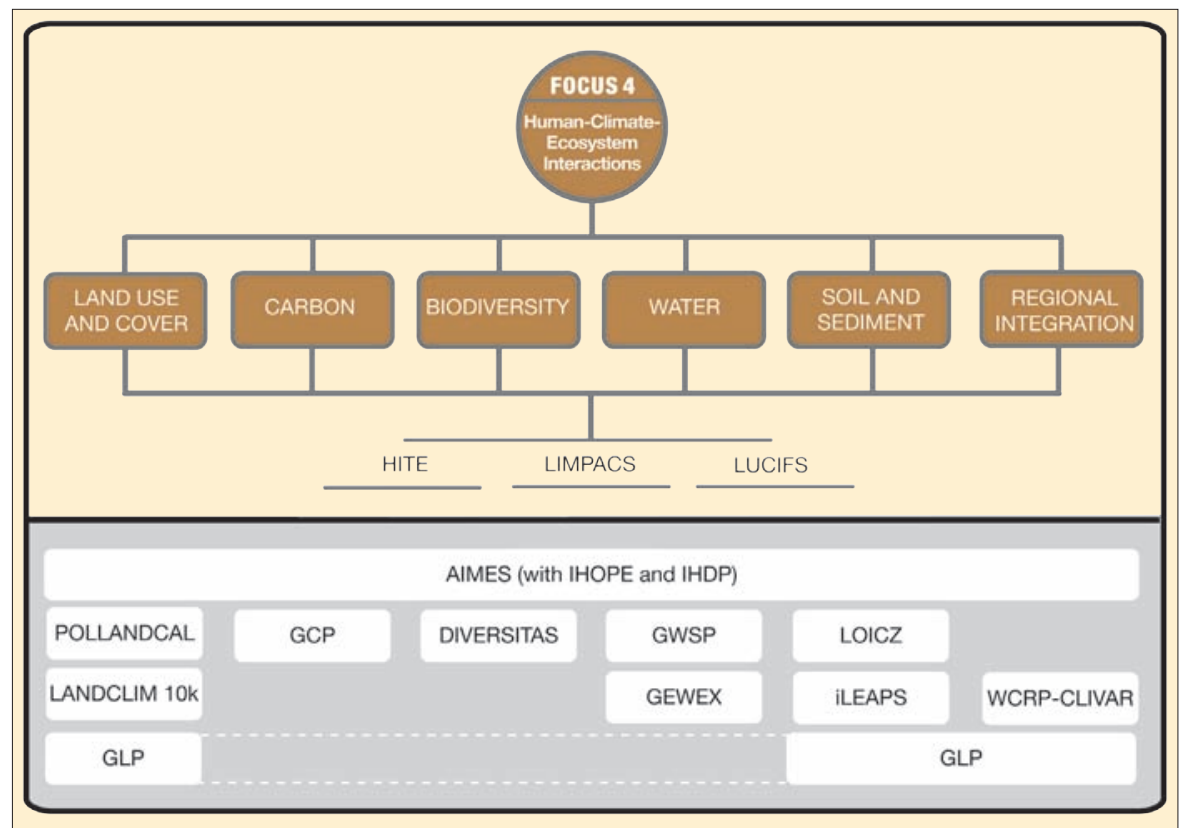

Figure 1: The new structure of PAGES Focus 4 (PHAROS) showing how the science undertaken within HITE, LIMPACS and LUCIFS communities contributes to major landscape elements through the activities of integration and modeling, with explicit links to ESSP/IGBP projects and other PAGES Foci and Cross-Cutting Themes. ture in ways that allowed explicit links to be made with other ESSP/IGBP projects and other PAGES Foci. At the heart of the new structure (Fig. 1) lie the key activities of Regional-Global Integration, applied to five major landscape elements (Carbon, Water, Soil and Sediment, Land Use and Cover, Biodiversity) through the combined activities of the HITE, LUCIFS and LIMPACS communities. Regions designated as Climate Change Hotspots are a priority under the Regional-Global Integration banner. The new structure enables more direct engagement with the activities and objectives of ESSP DIVERSITAS, ESSP Joint Projects Carbon and Water, and IGBP Core Projects AIMES, GLP, iLEAPS and LOICZ, particularly in terms of providing a temporal perspective for model development and validation.

\section{Acknowledgements}

We were pleased that so many scientists participated in the meeting, and would like to pay particular thanks to three postgraduates for their skills as meeting reporters - Alex Dickson, Emily Foster and Katy Wilson. 